\title{
TAFRO syndrome: current perspectives
}

This article was published in the following Dove Press journal:

Journal of Blood Medicine

\author{
Kentaro Sakashita ${ }^{1,2}$ \\ Kengo Murata ${ }^{2}$ \\ Mikio Takamori ${ }^{2}$ \\ 'Department of Internal Medicine, \\ Tokyo Metropolitan Matsuzawa \\ Hospital, ${ }^{2}$ Department of Respiratory \\ Medicine, Tokyo Metropolitan Tama \\ Medical Center, Tokyo, Japan
}

\begin{abstract}
Multicentric Castleman's disease (MCD), a distinct subtype of Castleman's disease, is a rare, nonneoplastic, lymphoproliferative disorder. Patients with MCD present with systemic symptoms and multiple lymphadenopathy. Lymph node biopsy is necessary for the diagnosis of various histological MCD patterns including hyaline vascular, plasma cell, and mixed types. Human herpesvirus 8 (HHV8) infection was identified as an important etiology of MCD among immunocompromised patients such as those positive for human immunodeficiency virus. Although HHV8-negative MCD was reported in immunocompetent patients, the underlying etiology remains unknown. Several experts speculate that MCD in immunocompetent patients might be due to proinflammatory hypercytokinemia because of infection by a virus other than HHV8, inflammation, or neoplastic disease. In 2010, a distinct variant of HHV8-negative MCD reported in Japan was characterized by thrombocytopenia, anasarca, myelofibrosis, renal dysfunction, and organomegaly (TAFRO). Recent case reports and a systematic review suggest that TAFRO syndrome might have a unique pathogenesis among HHV8-negative MCD variants. This review introduces TAFRO syndrome as a subtype of HHV8-negative MCD and offers an overview of the current perspectives on this syndrome.
\end{abstract}

Keywords: herpesvirus 8, human, multicentric Castleman's disease, interleukin-6

\section{Introduction}

Castleman's disease (CD), first reported in 1954, is a rare, nonneoplastic, lymphoproliferative disorder. ${ }^{1}$ Characteristic histopathological findings of CD are angiofollicular lymph node hyperplasia in a localized lymph node region. ${ }^{2}$ In 1974, Gaba et al described cases exhibiting this distinct histopathology in multiple lymph nodes and clinically classified CD into unicentric CD (UCD) and multicentric CD (MCD). ${ }^{3}$ Patients with UCD, who are usually younger than those with MCD, are often asymptomatic. Physical findings of UCD consist of enlargement of one lymph node with no radiologic evidence of lymphadenopathy in other areas. Conversely, patients with MCD have histologic findings of CD within at least one regional group of lymph nodes as well as clinical or radiologic evidence of additional lymphadenopathy. Patients with MCD show systemic symptoms stemming from reactive proliferation of benign lymphocytes. UCD is treated by surgical lymph node excision, which is curative for most patients. In contrast, systemic treatment is needed to control MCD. ${ }^{4,5} \mathrm{MCD}$ is further classified according to the patient's human herpesvirus 8 (HHV8) infection status. The histopathology of CD is classified into four variants: hyaline vascular (HV), plasma cell (PC), mixed, and plasmablastic. ${ }^{5,6}$ The plasmablastic variant is found only in patients with HHV8-associated MCD. Histological features of the HV variant include widened mantle zones composed of concentric
Correspondence: Kentaro Sakashita Department of Internal Medicine, Tokyo Metropolitan Matsuzawa Hospital, 2-I-I Kamikitazawa, Setagaya-ku, Tokyo I56-0057, Japan

$\mathrm{Tel} / \mathrm{fax}+813330372$ II

Email kensmile0524@gmail.com 
rings of small lymphocytes in an onion-skin pattern around small atrophic germinal centers with penetrating hyalinized vessels and dysplastic follicular dendritic cells (FDCs). ${ }^{7}$ In 2010, Takai et al proposed a new variant of HHV8-negative MCD characterized by thrombocytopenia, anasarca, myelofibrosis, renal dysfunction, and organomegaly and termed these presentations as thrombocytopenia, anasarca, myelofibrosis, renal dysfunction, and organomegaly (TAFRO) syndrome. ${ }^{8}$ We herein review TAFRO syndrome as a novel clinical entity in HHV8-negative MCD patients, which represents a group of systemic inflammatory disorders derived from an autoimmune pathophysiology.

\section{Clinical and histopathological features of MCD}

MCD is a distinct subtype of CD presenting with multiple lesions and systemic symptoms. Clinical features of MCD are systemic inflammation, reactive proliferation of benign lymphocytes, multicentric lymphadenopathy, polyclonal hypergammaglobulinemia, microcytic anemia, hypoalbuminemia, and elevated serum inflammatory proteins such as C-reactive protein (CRP) ${ }^{7,9} \mathrm{MCD}$ diagnosis is histologically confirmed by lymph node biopsy. These clinical and histopathological abnormalities might reflect hypercytokinemia based on unknown etiologies.

Lymph node enlargement with distinctive histological features characterizes MCD. As with $\mathrm{CD}$, the histological patterns of MCD include HV, PC, and mixed types. The HV type is the more common MCD variant, whereas the PC type mostly involves mediastinum. ${ }^{6}$ However, all lymph node-bearing regions and even tissues other than lymph nodes can be involved. The HV type is characterized by the proliferation of small, morphologically distinctive follicles that obscure the underlying nodal architecture (Figure 1). The follicles are surrounded by roughly concentric circular layers resembling onion skin which are composed of small lymphocytes in the mantle zone, with circularly arranged capillaries penetrating the germinal centers; ${ }^{10}$ these structures contain hyaline deposits and display a characteristic whorled appearance. In contrast, the interfollicular stromal tissue demonstrates prominent proliferation of capillaries and contains many small lymphocytes and PCs. Germinal centers are more typical of reactive follicular hyperplasia.

\section{HHV8 status and current classification of MCD}

Human immunodeficiency virus (HIV) infection has been recognized as an important risk factor for $\mathrm{MCD}$, and all

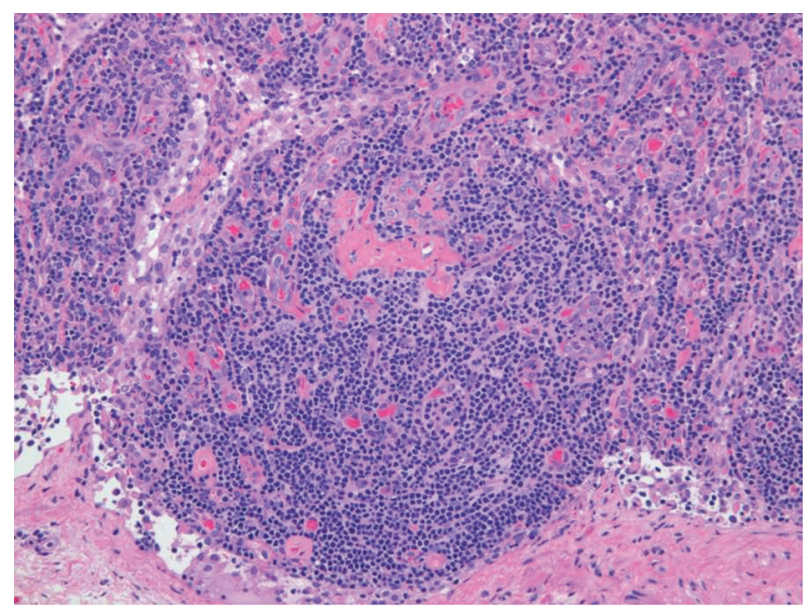

Figure I Histopathological features of hyaline-vascular type in a patient with multicentric Castleman's disease. Left inguinal node biopsy shows atrophic germinal centers and expansion of the interfollicular zone with vascular proliferation (hematoxylin and eosin stain).

patients with HIV-associated MCD are coinfected with HHV 8. Approximately $50 \%$ of HIV-negative MCD cases are infected with HHV8, which varies with the HHV8 prevalence in the population. ${ }^{11}$ Among patients with HHV8-associated MCD, HIV infection or another cause of immune compromise enables HHV8 to replicate in lymph nodes and to release human interleukin (IL)-6 and several other proinflammatory factors derived from HHV8. ${ }^{12,13}$ These proinflammatory factors induce proliferation of B lymphocytes and PCs, secretion of vascular endothelial growth factor (VEGF), which promotes angiogenesis, and an acute phase reaction. ${ }^{14}$ Plasma titers of HHV8 correlate with clinical symptoms and predict relapse rates in HIV-associated MCD. ${ }^{15} \mathrm{HHV} 8$ infection is responsible for MCD etiology in both HIV-positive and HIV-negative patients. Therefore, traditional HIV-based classification is not appropriate for identifying factors driving MCD pathogenesis. Fajgenbaum et al recently proposed a taxonomy to distinguish MCD on the basis of the HHV8 status, which provided a more accurate description of the pathogenesis and response to treatment (Figure 2). ${ }^{16}$

\section{HHV8-negative MCD (idiopathic MCD [iMCD])}

The presence of a subset of HHV8-negative MCD cases has long been acknowledged. Fajgenbaum et al proposed the term iMCD for HHV8-negative MCD. ${ }^{16}$ Although the etiology of iMCD remains unclear, several experts suggest that proinflammatory hypercytokinemia due to viral infection, inflammation, or neoplastic disease might be a mechanism. ${ }^{17,18}$ Fajgenbaum et al also proposed that one or more of the following three candidate processes might be 


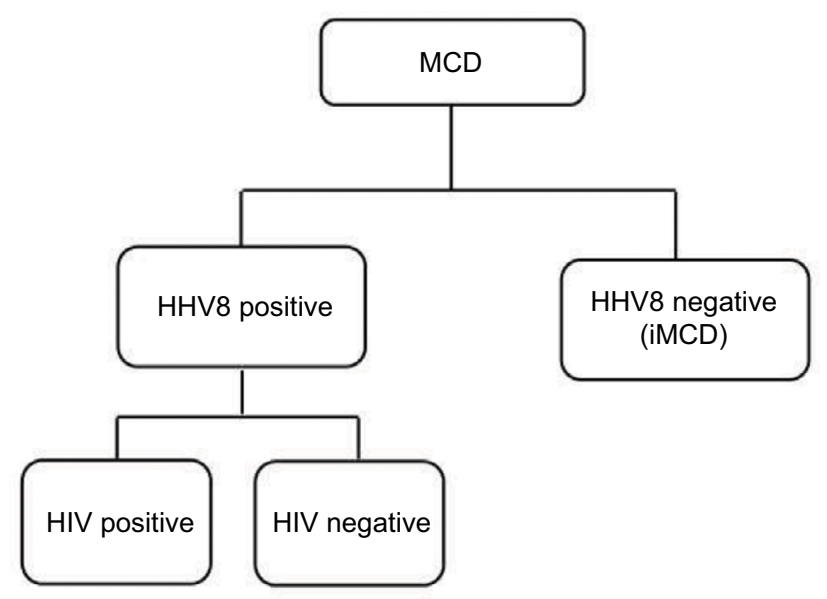

Figure 2 Proposed classification distinguishes multicentric Castleman's disease on the basis of human herpesvirus 8 status.

Note: Republished with permission of American Society of Hematology, from HHV8-negative, idiopathic multicentric Castleman disease: novel insights into biology, pathogenesis, and therapy, Fajgenbaum DC, Van Rhee F, Nabel CS, Blood, 123(19), 2014 copyright; permission conveyed through Copyright Clearance Center, Inc. ${ }^{16}$ Abbreviations: HHV8, human herpesvirus 8; HIV, human immunodeficiency virus; iMCD, idiopathic multicentric Castleman's disease.

responsible for hypercytokinemia in iMCD: ${ }^{16}$ autoimmune mechanisms by autoantibody-mediated antigenic stimulation or a germ-line genetic aberration in innate immune system (systemic inflammatory disease hypothesis), ectopic cytokine secretion by benign or malignant tumor cells within lymph nodes or extranodal tissue (paraneoplastic syndrome hypothesis), and viral signaling by a non-HHV8 virus (virally driven hypothesis).

\section{Introduction of TAFRO syndrome as an iMCD subtype}

Takai et al proposed a new variant of iMCD, whose features included TAFRO in their reported case series and named this variant TAFRO syndrome after its characteristic presentation. ${ }^{8}$ Lymph node biopsies of these cases demonstrated HV-type-like changes consistent with MCD. These patients with TAFRO features responded well to immunosuppressive therapy with glucocorticoids and cyclosporine. They considered this novel clinical entity of iMCD to represent a group of systemic inflammatory disorders with roots in autoimmune pathophysiology, on the basis of their favorable response to immunosuppressive therapy. ${ }^{19}$

\section{Clinical features of iMCD}

Liu et al published a systematic review of the literature on iMCD, providing information on its clinical features, treatment, and outcomes. ${ }^{20}$ Eighty-four articles fit all the inclusion criteria, and 128 patients were included in the review.
They showed that the clinical features of iMCD included systemic lymphadenopathy (128/128); anemia (79/91); elevated CRP (65/79); hypergammaglobulinemia (63/82); hypoalbuminemia (57/63); elevated IL-6 (57/63); hepatomegaly or splenomegaly (52/67); fever (33/64); edema, ascites, anasarca, or a combination of edema and anasarca (29/37); elevated soluble IL-2 receptor (20/21); and elevated VEGF (16/20). First-line treatment for iMCD included corticosteroids (47/128 [37\%]), cytotoxic chemotherapy (47/128 [37\%]), and anti-IL-6 therapy (11/128 [9\%]). The side effect profiles revealed that anti-IL-6 therapy demonstrated better tolerability than cytotoxic agents. Anti-IL-6 might require long-term administration because relapses were reported following cessation. ${ }^{21}$ The systematic review also showed that 49 of 116 patients (42\%) failed first-line therapy, 2-year survival was $88 \%$ (95\% confidence interval [CI] 81-95; 114 patients, 12 events, 36 events censored), and 27 of $121(22 \%)$ patients died by the end of the observed follow-up period (median, 29 months; interquartile range, 12-50 months). Factors negatively associated with survival reportedly included age ( $>37$ years), extravascular fluid overload, PC-type histopathology, TAFRO features, hypergammaglobulinemia, and thrombocytopenia. The 2-year survival rate was $85 \%$ (95\%CI 71-100) for iMCD patients with TAFRO features versus $92 \%$ (95\%CI 85-99) for those without TAFRO features (hazard ratio $2.67,95 \% \mathrm{CI}$ $0.4-11.20 ; p=0.16$ ). The increase in mortality among patients with TAFRO syndrome within the first 6 months of diagnosis is notable because it might reflect a highly aggressive phenotype. In addition, 24 of 128 patients (19\%) with iMCD had a diagnosis of malignant disease, a frequency significantly higher than that in the age-matched controls $(6 \%)$. Liu et $\mathrm{al}^{20}$ suggested several possible explanations for the increased prevalence of malignancies in iMCD. First, malignant cells might secrete IL-6 and other proinflammatory cytokines, leading to the histopathological and clinical features of iMCD. Second, iMCD might be a premalignant disease that eventually transforms into a malignancy. Third, an unknown genetic mutation might render the patient susceptible to both iMCD and malignant diseases. Fourth, excessive cytokine release might induce malignant transformation. Fifth, treatment modalities for iMCD such as cytotoxic therapy might amplify susceptibility to malignant disease. Finally, an unidentified virus might cause both $\mathrm{MCD}$ and the malignant disease. ${ }^{16}$ The authors suggested that clinicians should search for underlying malignant diseases in all patients who were newly diagnosed with iMCD and monitor those with iMCD for the development of malignant diseases. 


\section{Clinical and pathological presentation of TAFRO syndrome}

Iwaki et al analyzed the clinical features and pathological characteristics of iMCD in 23 cases from Japan and two cases from the USA. ${ }^{22}$ In their review, the authors categorized iMCD into two subtypes: iMCD with TAFRO symptoms (TAFROiMCD) and iMCD-not otherwise specified (iMCD-NOS) and found that TAFRO-iMCD was characterized by a more aggressive clinical course, refractoriness to corticosteroid therapy, more severe thrombocytopenia, higher frequency of anasarca, elevated levels of alkaline phosphatase, and almost normal gammaglobulin levels, compared with iMCD-NOS. These unique clinical and laboratory features suggested TAFRO-iMCD as a distinct variant of iMCD. This case series showed that IL-6 might not be a critical cytokine in TAFRO-iMCD, given that its levels were only modestly elevated in most cases of TAFRO-iMCD. Furthermore, the clinical features associated with elevated IL-6, such as thrombocytosis and polyclonal hypergammaglobulinemia, were not observed in TAFRO-iMCD cases. This finding contrasts with that of the control cases of iMCD-NOS. TAFRO-iMCD cases demonstrated thrombocytopenia with hyperplasia or normoplasia of megakaryocytes in the bone marrow at the time of diagnosis, possibly owing to increased peripheral thrombocyte consumption. Before the concepts of iMCD and TAFRO syndrome were established, Kojima et al classified Japanese MCD cases into two variants: idiopathic plasmacytic lymphadenopathy with polyclonal hyperimmunoglobulinemia (IPL) type and non-IPL type. ${ }^{23}$ Non-IPL MCD cases showed HV-type features, proliferation of vascularity in the interfollicular zone, and a higher incidence of pleural effusion and/or ascites, thrombocytopenia, and autoimmune diseases. They also reported that non-IPL cases exhibited FDC networks with expanded or disrupted follicles, which were observed in the TAFRO-iMCD group in the study by Iwaki et al. ${ }^{22}$ Conversely, IPL MCD cases exhibited clinical and pathological features more consistent with those of the iMCD-NOS group. On the basis of these findings, they speculated that TAFRO-iMCD might be immunological in origin. HHV8 status is not the sole basis of classification for MCD. Moreover, iMCD may be further categorized into iMCD with TAFRO features or iMCD-NOS as Iwaki et al demonstrated in their case series. ${ }^{22}$ Their proposed diagnostic criteria for iMCD with TAFRO features (Table 1) require fulfillment of the histopathological criteria, all major criteria, and one or more of the minor criteria. Diseases that had to be excluded to reach a definitive diagnosis included rheumatological diseases such as systemic lupus erythematosus; infectious diseases
Table I Proposed diagnostic criteria for TAFRO-iMCD

I. Histopathological criteria
- Compatible with the pathological findings of lymph nodes as
TAFRO-iMCD
- Negative LANA-I for HHV8
2. Major criteria: 3 of 5 TAFRO symptoms
I. Thrombocytopenia
II. Anasarca
III. Fever
IV. Reticulin fibrosis
V. Organomegaly
Absence of hypergammaglobulinemia
Small volume lymphadenopathy
3. One or more of the minor criteria
Hyperplasia/normoplasia of megakaryocytes in bone marrow
High serum level of ALP without markedly elevated serum transaminase Note: Republished with permission of American Journal of Hematology, Iwaki N, Fajgenbaum DC, Nabel CS, et al. Clinicopathologic analysis of TAFRO syndrome demonstrates a distinct subtype of HHV-8-negative multicentric Castleman disease. Am J Hematol. 2016;91 (2):220-226. @ 2015 Wiley Periodicals, Inc. ${ }^{22}$

Abbreviations: ALP, alkaline phosphatase; HHV8, human herpesvirus 8; iMCD, idiopathic multicentric Castleman's disease; LANA-I, latency-associated nuclear antigen-I;TAFRO, thrombocytopenia, anasarca, myelofibrosis, renal dysfunction, and organomegaly.

such as acute Epstein-Barr virus; and neoplastic diseases such as lymphoma, POEMS (polyneuropathy, organomegaly, endocrinopathy, monoclonal gammopathy, and skin changes) syndrome, and other cancers. The characteristic lymph node findings in TAFRO-iMCD included atrophic germinal centers with enlarged endothelial cell nuclei, proliferation of endothelial venules with an enlarged nucleus in the interfollicular zone, and a small number of mature PCs.

\section{Approach to establishing diagnostic criteria for TAFRO syndrome in Japan}

Kawabata et al proposed an MCD subtype called CastlemanKojima disease, which they defined as a systemic inflammatory disorder in patients with HHV8-negative MCD in the absence of any known autoimmune or lymphoproliferative diseases. ${ }^{24}$ Castleman-Kojima disease can involve bone marrow, pleura, peritoneum, kidneys, liver, or lymph nodes. They also suggested that non-IPL MCD encompassed Castleman-Kojima disease, IgG4-related disease, POEMS syndrome, and malignant lymphomas. ${ }^{24}$ Table 2 shows the criteria proposed for the diagnosis of Castleman-Kojima disease, which can also be used as the diagnostic criteria for TAFRO syndrome. ${ }^{24}$

Iwaki et al attempted to develop a precise definition of TAFRO symptoms in their clinicopathological review of 25 cases. In that review, thrombocytopenia was defined as a platelet count $<100,000 / \mathrm{mm}^{3}$ at the time of diagnosis. Fever was defined as a temperature $>38.0^{\circ} \mathrm{C}\left(100.4^{\circ} \mathrm{F}\right)$. Anasarca 
Table 2 Definition of Castleman-Kojima disease (TAFRO syndrome)

Blood count abnormalities: low platelet and/or red blood cell count Thrombocytopenia

Microcytic anemia

Systemic inflammation

Polyserositis (pleuritis/peritonitis), inflammation of the tissue lining the lungs or abdominal cavities

Pleural effusions

Ascites

Renal dysfunction

Myelofibrosis

Immunological disorders

Rheumatoid factor, platelet-associated lgG, antithyroid antibody, and positivity on direct

Coombs test

Antinuclear antibody

Rare polyclonal hypergammaglobulinemia: $<4.0 \mathrm{~g} / \mathrm{L}$

Laboratory data abnormalities

Elevated level of alkaline phosphatase and decreased level of lactate dehydrogenase

Elevated levels of interleukin- 6 and the vascular endothelial growth factor in serum or effusion

Lymphadenopathy

Generally mild $(<1.5 \mathrm{~cm}$ in diameter)

Histology of mixed-type and less frequently HV-type $C D$

Abbreviations: CD, Castleman's disease; HV, hyaline vascular; TAFRO, thrombocytopenia, anasarca, myelofibrosis, renal dysfunction, and organomegaly.

was defined as the presence of pleural fluid and ascites by computed tomography (CT). Organomegaly included lymphadenopathy, hepatomegaly, and splenomegaly, which were confirmed by the evaluation of the CT scan by a radiologist. Reticulin fibrosis was assessed by bone marrow biopsy. ${ }^{22}$

\section{The first international consensus diagnostic criteria for iMCD}

The lack of established diagnostic criteria or disease-specific biomarkers for iMCD might hinder clinicians from reaching the correct diagnosis and starting treatment before the development of organ dysfunction and death. Clinical and pathological diagnostic criteria are urgently needed to facilitate disease recognition, diagnostic evaluation, and research on pathogenesis and treatment. In 2012, Fajgenbaum et al assembled a group of physicians, researchers, and patients to create the Castleman Disease Collaborative Network $(\mathrm{CDCN})$, with the aim to accelerate research through a targeted, collaborative, and patient-centric approach. ${ }^{25}$ The CDCN scientific advisory board assembled clinical data for 244 iMCD patients and 88 lymph node tissue biopsies for histopathologic review, including 128 cases analyzed in a systematic review of the literature on pathology-based iMCD. ${ }^{20}$ Thirty-seven cases were submitted by the CDCN working group members, and 79 cases derived from a randomized controlled study of the anti-IL-6 chimeric monoclonal antibody siltuximab in subjects with symptomatic iMCD. ${ }^{26} \mathrm{An}$ international symposium sponsored by the $\mathrm{CDCN}$ and the University of Pennsylvania Orphan Disease Center was held in 2015 to establish the diagnostic criteria for iMCD. All votes were anonymous, and $75 \%$ agreement was needed to pass an individual decision. The final criteria vote required $100 \%$ consensus. On the basis of the consensus at this symposium, Fajgenbaum et al recently published the multidisciplinary, evidence-based, diagnostic criteria for iMCD. ${ }^{27}$ The three-part criteria were finally accepted by the working group (Table 3 ). A patient must meet both major criteria and fulfill at least two of the 11 minor criteria including at least one laboratory abnormality for a diagnosis of $\mathrm{MMCD}$. In addition, diseases listed in the exclusion criteria must be ruled out.

\section{Treatment for iMCD and TAFRO syndrome Corticosteroids}

Corticosteroids are immunosuppressive drugs that treat both acute and chronic inflammation by decreasing transcription of proinflammatory cytokines and chemokines, adhesion molecules, and key enzymes in the inflammatory process such as IL-2, IL-6, and tumor necrosis factor- $\alpha$. Corticosteroids are used as first-line treatment for iMCD including TAFRO syndrome. ${ }^{28}$ However, patients frequently relapse, and corticosteroids are usually used in combination with other drugs. Nonresponders to corticosteroids are usually treated with various alternative drugs including anti-IL-6 and the chemotherapeutic agents anakinra, bortezomib, and thalidomide. However, there are limited data on prognosis and response to these treatments.

\section{Anti-IL-6 therapies Siltuximab}

Siltuximab is a human-murine chimeric monoclonal antibody that binds to IL-6 with high affinity. A Phase III clinical trial suggested that siltuximab and optimal supportive care were superior to optimal supportive care alone and that the drug was well tolerated even during prolonged administration. Siltuximab also had a favorable effect on radiological response in lymph nodes, anemia, other disease symptoms, and inflammatory parameters. Furthermore, siltuximab therapy was not associated with a significant increase in adverse events compared with placebo. ${ }^{26}$ However, siltuximab is approved for the treatment of MCD only in North America and Europe. 
Table 3 Consensus diagnostic criteria for iMCD

I. Major criteria (both I and 2 are needed)

I. Histopathological lymph node features consistent with the iMCD spectrum (Figure 3). Features along the iMCD spectrum include (grades 2-3 for either regressive GCs or plasmacytosis is required at a minimum): regressed/atrophic/atretic germinal centers, often with expanded mantle zones composed of concentric rings of lymphocytes in an "onion-skin" appearance, FDC prominence, vascularity, often with prominent endothelium in the interfollicular space and vessels penetrating into the GCs with a "Iollipop" appearance, sheet-like, polytypic plasmacytosis in the interfollicular space, hyperplastic GCs

2. Enlarged lymph nodes ( $\geq 1 \mathrm{~cm}$ in short-axis diameter) in $\geq 2$ lymph node stations

II. Minor criteria (at least 2 of II criteria and at least I laboratory criterion are needed)

Laboratory

I. Elevated CRP (>10 mg/L) or ESR ( $>15 \mathrm{~mm} / \mathrm{h})$

2. Anemia (hemoglobin, $125 \mathrm{~g} / \mathrm{L}$ for male and $<115 \mathrm{~g} / \mathrm{L}$ for female)

3. Thrombocytopenia (platelet count $<150 \mathrm{k} / \mathrm{mL}$ ) or thrombocytosis (platelet count $<400 \mathrm{k} / \mathrm{mL}$ )

4. Hypoalbuminemia (albumin $<35 \mathrm{~g} / \mathrm{L}$ )

5. Renal dysfunction (eGFR, $60 \mathrm{~mL} / \mathrm{min} / 1.73 \mathrm{~m}^{2}$ ) or proteinuria (total protein $150 \mathrm{mg} / 24 \mathrm{~h}$ or $10 \mathrm{mg} / 100 \mathrm{~mL}$ )

6. Polyclonal hypergammaglobulinemia (total g globulin or immunoglobulin G. I.7 g/L)

Clinical

I. Constitutional symptoms: night sweats, fever $\left(>38^{\circ} \mathrm{C}\right)$, weight loss, or fatigue ( $\geq 2$ CTCAE lymphoma score for B-symptoms)

2. Large spleen and/or liver

3. Fluid accumulation: edema, anasarca, ascites, or pleural effusion

4. Eruptive cherry hemangiomatosis or violaceous papules

5. Lymphocytic interstitial pneumonitis

III.Exclusion criteria (must rule out all diseases that can mimic iMCD)

Infection-related disorders

I. HHV8 (infection can be documented by blood PCR; diagnosis of HHV8-associated MCD requires positive LANA-I staining by IHC, which excludes iMCD)

2. Clinical EBV-lymphoproliferative disorders such as infectious mononucleosis or chronic active EBV (detectable EBV viral load not necessarily exclusionary)

Autoimmune/autoinflammatory diseases

(requires full clinical criteria; detection of autoimmune antibodies alone is not exclusionary)

I. Systemic lupus erythematosus

2. Rheumatoid arthritis

3. Adult-onset Still's disease

4. Juvenile idiopathic arthritis

Autoimmune lymphoproliferative syndrome and malignant/lymphoproliferative disorders (these disorders must be diagnosed before or at the same time as iMCD to be exclusionary):

I. Lymphoma (Hodgkin and non-Hodgkin)

2. Multiple myeloma

3. Primary lymph node plasmacytoma

4. FDC sarcoma

5. POEMS syndrome

Inflammation and adenopathy caused by other uncontrolled infections

(eg, acute or uncontrolled CMV, toxoplasmosis, HIV, and active tuberculosis)

Note: Republished with permission of American Society of Hematology, from International, evidencebased consensus diagnostic criteria for HHV-8 - negative/idiopathic multicentric Castleman disease, Fajgenbaum DC, Uldrick TS, Bagg A, et a, Blood, I29(12), 2017, permission conveyed through Copyright Clearance Center, Inc. ${ }^{27}$

Abbreviations: CMV, cytomegalovirus; CRP, C-reactive protein; CTCAE, common terminology for adverse events; EBV, Epstein-Barr virus; eGFR, estimated glomerular filtration rate; ESR, erythrocyte sedimentation rate; FDC, follicular dendritic cell; GC, germinal center; HHV8, human herpesvirus 8; HIV, human immunodeficiency virus; iMCD, idiopathic multicentric Castleman's disease; IHC, immunohistochemistry; LANA-I, latency-associated nuclear antigen; PCR, polymerase chain reaction; POEMS, polyneuropathy, organomegaly, endocrinopathy, monoclonal gammopathy, and skin changes.

\section{Tocilizumab}

Tocilizumab is a humanized IL-6 antagonist capable of blocking transmembrane signaling of IL- $6 .{ }^{29}$ It reduces inflammation related to the IL-6 signaling cascade. Tocilizumab is currently approved for the treatment of MCD in Japan and rheumatoid arthritis worldwide. ${ }^{30}$

\section{Thalidomide}

Thalidomide is an immune modulator that inhibits tumor necrosis factor- $\alpha$, IL-1, IL-6, IL-12, and VEGF, while also stimulating T cells. ${ }^{31}$ Thalidomide has demonstrated efficacy in inducing remission, decreasing IL-6 levels, and lowering CRP in patients with iMCD. ${ }^{32,33}$

\section{Anti-CD20 therapies Rituximab}

Rituximab is a chimeric monoclonal antibody that binds to CD20, which is approved for the treatment of non-Hodgkin lymphoma. Rituximab is frequently used as first- or secondline therapy for MCD. One case report suggested that 


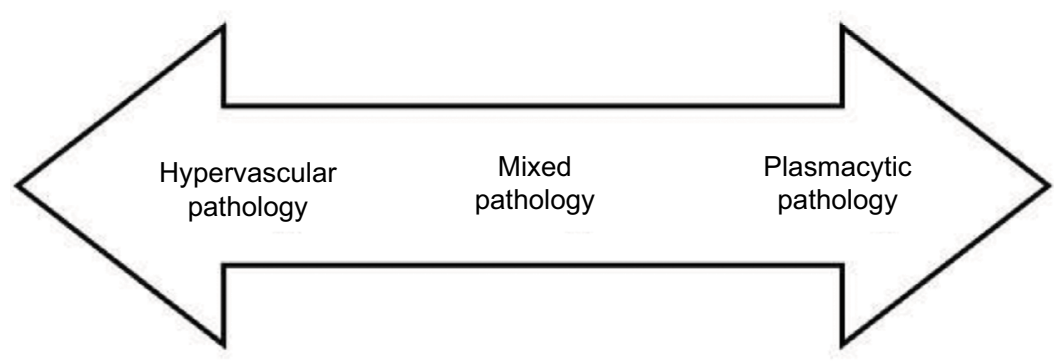

\begin{tabular}{|l|}
\hline A: Regressed germinal centers \\
\hline B: FDC prominence \\
\hline C: Vascularity \\
\hline D: Hyperplastic germinal centers \\
\hline E: Plasmacytosis \\
\hline
\end{tabular}

Figure 3 Histopathologic features of Castleman's disease. Hypervascular subtype is characterized by the presence of regressed germinal centers and prominent FDC, whereas the plasmacytic subtype exhibits hyperplastic germinal centers and profuse plasmacytosis. The mixed subtype exhibits a combination of hypervascular and plasmocytic features. Vascularity is frequently observed in idiopathic multicentric Castleman's disease but can be seen in either subtype as well. Republished with permission of American Society of Hematology, from International, evidencebased consensus diagnostic criteria for HHV-8 - negative/idiopathic multicentric Castleman disease, Fajgenbaum DC, Uldrick TS, Bagg A, et a, Blood, I29(12), 2017, permission conveyed through Copyright Clearance Center, Inc. ${ }^{27}$

Abbreviation: FDC, follicular dendritic cell.

rituximab might be a therapeutic option for iMCD cases associated with immune-related disorders. ${ }^{34}$ However, rituximab might be only partially effective and does not provide longterm remission if the treatment is initiated early in the course of disease before poor performance status scores decline. ${ }^{35}$

\section{Cytotoxic chemotherapy}

Cytotoxic, lymphoma-based chemotherapeutic agents including cyclophosphamide, doxorubicin, vincristine, and prednisone induce responses in a large portion of the severely ill iMCD patients; however, relapses are common, and the side effects are significant. ${ }^{36}$

\section{Bortezomib}

Recently, therapeutic approaches to target pathways upstream of IL-6 in iMCD have been reported, which need further evaluation, particularly for anti-IL-6 therapy-refractory patients. Bortezomib, a selective proteasome inhibitor that preferentially targets PCs, was reported to lower IL-6 levels and induce remission in four iMCD cases. ${ }^{37,38}$ Bortezomib might work in iMCD via direct inhibition of nuclear factorkappa B by degrading the IkB kinase. ${ }^{39}$

\section{Future perspectives on TAFRO syndrome in iMCD}

Recent case series and systematic reviews demonstrated that $\mathrm{iMCD}$ and TAFRO syndrome include heterogeneous diseases driven by pathological hypercytokinemia due to an unknown etiology, as previously mentioned. Although the etiology and the appropriate treatment strategies remain elusive, TAFRO syndrome might have a unique pathogenesis distinct from that of iMCD-NOS. For instance, the case series by Iwaki et al showed little evidence of an association between serum IL-6 concentration and the efficacy of anti-IL-6 therapy in TAFRO syndrome, suggesting that an elevated serum IL-6 level might not be the primary pathogenesis driving proinflammatory hypercytokinemia. ${ }^{22}$ Furthermore, a clinicopathological review suggested that none of the TAFRO-iMCD cases demonstrated hypergammaglobulinemia, whereas almost all iMCD-NOS cases exhibited polyclonal hypergammaglobulinemia. Therefore, different therapeutic approaches are needed for TAFRO patients. To facilitate early diagnosis and treatment, a precise classification of the iMCD subtypes is necessary.

Recent studies have shed more light on TAFRO syndrome. First, two large systematic reviews clearly characterized the clinical manifestations and pathological findings of iMCD and TAFRO. ${ }^{20,22}$ Second, an international consensus on the diagnostic criteria has been established by the CDCN leadership, which further clarified the laboratory and clinical features of MCD using a global patient registry and case reports. ${ }^{27}$ The etiology of iMCD awaits a similar clarification to improve disease management and treatment outcomes. 


\section{Conclusion}

TAFRO syndrome is a recently established, distinct variant of HHV8-negative MCD with an autoimmune etiology. The characteristics include more aggressive clinical course, more severe thrombocytopenia, higher frequency of anasarca, and almost normal serum gammaglobulin levels compared with those of non-TAFRO iMCD. The recently established international consensus diagnostic criteria for iMCD will accordingly facilitate the identification of this syndrome. Further studies are needed to establish the clinical and pathological features of TAFRO syndrome by identifying biomarkers, pathophysiology, etiology, and treatment strategies based on evidence.

\section{Disclosure}

The authors report no conflicts of interest in this work.

\section{References}

1. Castleman B, Iverson L, Menendez VP. Localized mediastinal lymphnode hyperplasia resembling thymoma. Cancer. 1956;9(4):822-830.

2. Castleman B, Towne VW. Case records of the Massachusetts General Hospital; weekly clinicopathological exercises; founded by Richard C. Cabot. N Engl J Med. 1954;251(10):396-400.

3. Gaba AR, Stein RS, Sweet DL, Variakojis D. Multicentric giant lymph node hyperplasia. Am J Clin Pathol. 1978;69(1):86-90.

4. Bowne WB, Lewis JJ, Filippa DA, et al. The management of unicentric and multicentric Castleman's disease: a report of 16 cases and a review of the literature. Cancer. 1999;85(3):706-717.

5. Waterston A, Bower M. Fifty years of multicentric Castleman's disease. Acta Oncol. 2004;43(8):698-704.

6. Keller AR, Hochholzer L, Castleman B. Hyaline-vascular and plasmacell types of giant lymph node hyperplasia of the mediastinum and other locations. Cancer. 1972;29(3):670-683.

7. Cronin DMP, Warnke RA. Castleman disease - an update on classification and the spectrum of associated lesions. Adv Anat Pathol. 2009;16(4):236-246.

8. Takai K, Nikkuni K, Shibuya H, Hashidate H. [Thrombocytopenia with mild bone marrow fibrosis accompanied by fever, pleural effusion, ascites and hepatosplenomegaly]. Rinsho Ketsueki. 2010;51(5):320-325. Japanese.

9. Kawabata H, Kadowaki N, Nishikori M, et al. Clinical features and treatment of multicentric Castleman's disease: a retrospective study of 21 Japanese patients at a single institute. J Clin Exp Hematop. 2013;53(1):69-77.

10. Shahidi H, Myers JL, Kvale PA. Castleman's disease. Mayo Clin Proc. 1995;70(10):969-977.

11. Soumerai JD, Sohani AR, Abramson JS. Diagnosis and management of Castleman disease. Cancer Control. 2014;21(4):266-278.

12. Dossier A, Meignin V, Fieschi C, Boutboul D, Oksenhendler E, Galicier L. Human herpesvirus 8-related Castleman disease in the absence of HIV infection. Clin Infect Dis. 2013;56(6):833-842.

13. Leroy S, Moshous D, Cassar O, et al. Multicentric Castleman disease in an HHV8-infected child born to consanguineous parents with systematic review. Pediatrics. 2012;129(1):e199-e203.

14. Kishimoto T. IL-6: from its discovery to clinical applications. Int Immunol. 2010;22(5):347-352.

15. Stebbing J, Adams C, Sanitt A, et al. Plasma HHV8 DNA predicts relapse in individuals with HIV-associated multicentric Castleman disease. Blood. 2011;118(2):271-275.

16. Fajgenbaum DC, Van Rhee F, Nabel CS. HHV-8-negative, idiopathic multicentric Castleman disease: novel insights into biology, pathogenesis, and therapy. Blood. 2014;123(19):2924-2933.
17. Suda T, Katano H, Delsol G, et al. HHV-8 infection status of AIDSunrelated and AIDS-associated multicentric Castleman's disease. Pathol Int. 2001;51(9):671-679.

18. Dossier A, Meignin V, Fieschi C, Boutboul D, Oksenhendler E, Galicier L. Human herpesvirus 8-related Castleman disease in the absence of HIV infection. Clin Infect Dis. 2013;56(6):833-842.

19. Takai K, Nikkuni K, Momoi A, Nagai K, Igarashi N, Saeki T. Thrombocytopenia with reticulin fibrosis accompanied by fever, anasarca and hepatosplenomegaly: a clinical report of five cases. J Clin Exp Hematop. 2013;53(1):63-68.

20. Liu AY, Nabel CS, Finkelman BS, et al. Idiopathic multicentric Castleman's disease: a systematic literature review. Lancet Haematol. 2016;3(4):e163-e175.

21. Nishimoto N, Sasai M, Shima Y, et al. Improvement in Castleman's disease by humanized anti-interleukin- 6 receptor antibody therapy. Blood. 2000;95(1):56-61.

22. Iwaki N, Fajgenbaum DC, Nabel CS, et al. Clinicopathologic analysis of TAFRO syndrome demonstrates a distinct subtype of HHV-8-negative multicentric Castleman disease. Am J Hematol. 2016;91(2):220-226.

23. Kojima M, Nakamura N, Tsukamoto N, et al. Clinical implications of idiopathic multicentric Castleman disease among Japanese: a report of 28 cases. Int J Surg Pathol. 2008;16(4):391-398.

24. Kawabata H, Takai K, Kojima M, et al. Castleman-Kojima disease (TAFRO syndrome): a novel systemic inflammatory disease characterized by a constellation of symptoms, namely, thrombocytopenia, ascites (anasarca), microcytic anemia, myelofibrosis, renal dysfunction, and organomegaly: a status. J Clin Exp Hematop. 2013;53(1):57-61.

25. Fajgenbaum DC, Ruth JR, Kelleher D, Rubenstein AH. The collaborative network approach: a new framework to accelerate Castleman's disease and other rare disease research. Lancet Haematol. 2016;3(4):e150-e152.

26. Van Rhee F, Wong RS, Munshi N, et al. Siltuximab for multicentric Castleman's disease: a randomised, double-blind, placebo-controlled trial. Lancet Oncol. 2014;15(9):966-974.

27. Fajgenbaum DC, Uldrick TS, Bagg A, et al. International, evidencebased consensus diagnostic criteria for HHV-8 - negative/idiopathic multicentric Castleman disease. Blood. 2017;129(12):1646-1658.

28. Frizzera G, Banks PM, Massarelli G, Rosai J. A systemic lymphoproliferative disorder with morphologic features of Castleman's disease. Pathological findings in 15 patients. Am J Surg Pathol. 1983;7(3): 211-231.

29. Sato K, Tsuchiya M, Saldanha J, Bendig MM. Reshaping a human antibody to inhibit the interleukin 6-dependent tumor cell growth. Cancer Res. 1993;53(4):851-856.

30. Kishimoto T. Interleukin-6: discovery of a pleiotropic cytokine. Arthritis Res Ther. 2006;8(Suppl 2):S2.

31. Lopez-Girona A, Mendy D, Ito T, et al. Cereblon is a direct protein target for immunomodulatory and antiproliferative activities of lenalidomide and pomalidomide. Leukemia. 2012;26(11):2326-2335.

32. Starkey CR, Joste NE, Lee F-C. Near-total resolution of multicentric Castleman disease by prolonged treatment with thalidomide. Am J Hematol. 2006;81(4):303-304.

33. Tatekawa S, Umemura K, Fukuyama R, et al. Thalidomide for tocilizumab-resistant ascites with TAFRO syndrome. Clin Case Reports. 2015;3(6):472-478.

34. Ocio EM, Sanchez-Guijo FM, Diez-Campelo M, et al. Efficacy of rituximab in an aggressive form of multicentric Castleman disease associated with immune phenomena. Am J Hematol. 2005;78(4):302-305.

35. Gholam D, Vantelon J-M, Al-Jijakli A, Bourhis J-H. A case of multicentric Castleman's disease associated with advanced systemic amyloidosis treated with chemotherapy and anti-CD20 monoclonal antibody. Ann Hematol. 2003;82(12):766-768.

36. Chronowski GM, Ha CS, Wilder RB, Cabanillas F, Manning J, Cox JD. Treatment of unicentric and multicentric Castleman disease and the role of radiotherapy. Cancer. 2001;92(3):670-676.

37. Hess G, Wagner V, Kreft A, Heussel CP, Huber C. Effects of bortezomib on pro-inflammatory cytokine levels and transfusion dependency in a patient with multicentric Castleman disease. Br J Haematol. 2006;134(5): 544-545. 
38. Sobas MA, Vence NA, Arias JD, Lopez AB, Rodriguez MF, Lopez JLB. Efficacy of bortezomib in refractory form of multicentric Castleman disease associated to poems syndrome (MCD-POEMS variant). Ann Hematol. 2010;89(2):217-219.
39. Juvekar A, Manna S, Ramaswami S, et al. Bortezomib induces nuclear translocation of IB resulting in gene-specific suppression of NF-Bdependent transcription and induction of apoptosis in CTCL. Mol Cancer Res. 2011;9(2):183-194.

\section{Publish your work in this journal}

The Journal of Blood Medicine is an international, peer-reviewed, open access, online journal publishing laboratory, experimental and clinical aspects of all aspect pertaining to blood based medicine including but not limited to: Transfusion Medicine; Blood collection, Donor issues, Transmittable diseases, and Blood banking logistics; Immunohematology; Artificial and alternative

\section{Dovepress}

blood based therapeutics; Hematology; Biotechnology/nanotechnology of blood related medicine; Legal aspects of blood medicine; Historical perspectives. The manuscript management system is completely online and includes a very quick and fair peer-review system. Visit http://www.dovepress.com/ testimonials.php to read real quotes from published authors.

Submit your manuscript here: http://www.dovepress.com/journal-of-blood-medicine-journa 